\title{
Intervention studies for improving global health and health care: An important arena for epidemiologists
}

\author{
G. Kvåle, H. Sommerfelt and K. Fylkesnes \\ Centre for International Health, University of Bergen, Norway \\ Correspondence: Gunnar Kvåle, Centre for International Health, Armauer Hansen Building, NO-5021 Bergen, Norway
}

\begin{abstract}
SUMMARY
Marginalised populations in many low- and middle-income countries experience an increasing burden of disease, in sub-Saharan Africa to a large extent due to faltering health systems and serious HIV epidemics. Also other poverty related diseases (PRDs) are prevalent, especially respiratory and diarrhoeal diseases in children, malnutrition, maternal and perinatal health problems, tuberculosis and malaria. Daily, nearly 30,000 children under the age of 5 die, most from preventable causes, and 8,000 people die from HIV infections. In spite of the availability of powerful preventive and therapeutic tools for combating these PRDs, their implementation, especially in terms of equitable delivery, leaves much to be desired. The research community must address this tragic gap between knowledge and implementation. Epidemiologists have a very important role to play in conducting studies on diseases that account for the largest share of the global disease burden. A shift of focus of epidemiologic research towards intervention studies addressing health problems of major public health importance for disadvantaged population groups is needed. There is a need to generate an evidence-base for interventions that can be implemented on a large scale; this can result in increased funding of health promotion programs as well as enable rational prioritization and integration between different health interventions. This will require close and synergetic teamwork between epidemiologists and other professions across disciplines and sectors. In this way epidemiologists can contribute significantly to improve health and optimise health care delivery for marginalized populations.
\end{abstract}

\section{INTRODUCTION}

During the last decades, basic and public health research have produced new technologies and knowledge on how to diagnose, treat and prevent diseases and to promote health. These measures have been successfully implemented in most affluent regions of the world, contributing to better health and health care. During the decades before 1990, substantial progress was also observed in most poor countries, with decreasing child mortality and increasing life expectancy. However, during the last decade, marginalised populations in many low- and middle-income countries have experienced an increasing burden of disease and disability, in sub-Saharan Africa to a large extent due to faltering health systems and serious HIV epidemics, which has caused a decrease in life expectancy of more than 10 years in many countries (1). Also, other PRDs remain prevalent, especially respiratory and diarrhoeal diseases in children, malnutrition, maternal and perinatal health problems, tuberculosis and malaria.

Currently, resource-poor countries are experiencing an influx of resources to some parts of the health system, through targeted funding from donor organizations and global initiatives e.g. the Global Fund to fight AIDS, Tuberculosis and Malaria, Global Alliance for Vaccines and Immunisation and other programmes targeting specific diseases. In particular, the recent influx of antiretroviral drugs and demand for treatment of AIDS are overstretching the capacity of health systems in many poor countries. In parallel to this resource influx, health systems functioning has deteriorated in many countries, with an accelerating "brain drain" (2) and an increasing disparity between policies and actual practices. Many introduced reforms have not been evidence-based and structural adjustment programmes, rapid decentralization and cost-sharing have in many areas caused disruption of even well-functioning services. A particularly unfortunate outcome of these reforms has been increasing inequity in health and access to health care (3), resulting in a resurgence of PRDs among large segments of poor populations. As a consequence, many countries, particularly in subSaharan Africa, are now struggling with increasing disease burdens, a collapsing health system and increasing inequalities in availability of effective health care (4).

Powerful tools are available for combating PRDs. However, in spite of the availability of effective preventive and therapeutic methods, their implementation on a large scale leaves much to be desired, especially in terms of equitable delivery and the attainment of the Millennium Development Goals (5). The implementation gap is partly due to lack of resources, in terms of funds, infrastructure and personnel, but is also due to a lack of knowledge of how these interventions may be better implemented within existing health systems, including how the systems need to be changed to enable 
the interventions to fulfil their potential. It is also due to socio-cultural factors affecting communities as well as health systems and their personnel, which may inhibit effective and appropriate prevention and treatment efforts.

In this paper we describe the role of epidemiologic research in the identification of effective interventions for PRDs and for improving implementation by addressing the problems of translating effective prevention and treatment interventions as well as health promotion activities into effective programmes in resourcepoor settings. As examples of areas of attention, we will describe the situation regarding common illnesses and malnutrition in children and HIV infection, that together account for nearly $50 \%$ of the disability adjusted life years lost in Africa, and the health systems factors of importance for prevention, treatment and care of PRDs in general.

\section{EXAMPLES OF IMPORTANT AREAS OF RESEARCH}

\section{Child Health and Nutrition}

In the period 2000-2003, only five causes accounted for about two thirds of the total number of deaths ( $\square 10.6$ million) among children under 5 years of age (U5): pneumonia (19\%), diarrhoea (18\%), and neonatal causes, such as severe infection $(10 \%)$, preterm delivery $(10 \%)$ and asphyxia at birth $(8 \%)$. More than half of these deaths are associated with undernutrition (6). Two thirds of these deaths could have been prevented by making known, cost-effective interventions (e.g. promotion of exclusive breast feeding and adequate complementary feeding, equitable delivery of existing vaccines, vitamin A supplementation and treatment of diarrhoea with zinc) universally available (7). Every year, an estimated four million babies die in the first four weeks of life, $70 \%$ of these deaths may be prevented with equitable and scaled up implementation of known interventions, such as tetanus immunization of pregnant women, eclampsia prevention, early initiation of breast feeding, and adequate perinatal care $(8,9)$. To achieve MDG 4 (to reduce U5 mortality by two thirds from 1990 to 2015), essential child health interventions must accordingly be provided where they are needed most and must reach the poor. A high proportion of the $\square 7$ million perinatal (8) and of the annual $\square 500.000$ maternal deaths could be prevented if known effective measures were implemented on a global scale, also among the poor segments of the population $(9,10)$.

\section{HIV infection}

Severe HIV epidemics are having a devastating impact on living conditions, child and adult mortality, the social/health sector and are undermining development in sub-Saharan Africa (11). As HIV seriously impairs productivity, it is both a major cause and consequence of poverty $(12,13)$. After 20 years of preventive efforts, the number of people infected with HIV continues to rise and there are still few examples of declining incidence of HIV infection. In Southern Africa, the prevalence has been stable in most areas since 1990 , and with levels of $25 \%$, as many as $90 \%$ of all deaths in the age-group 25-39 years are related to HIV infection (14). There is a growing realization that HIV prevention efforts too often have been built on broad categories of types of epidemics rather than knowledge on local transmission patterns and risk exposure. There are substantial differences in the HIV prevalence within and between countries in sub-Saharan Africa, and each epidemic will have a different dynamic and course (14-16). These differences are often important enough to require different preventive approaches, clearly underscoring the vital importance of understanding the local contexts. Health systems research has also revealed that the demand for potentially effective preventive programmes, such as voluntary testing, is critically low due to poor perception of the health service in general (17).

\section{Research on health systems factors}

Although there is still an obvious need to develop new interventions against PRDs, it is increasingly recognized that priority must also be given to research that can contribute in guiding health systems on how to deliver preventive and curative health services in an equitable and acceptable way. There is insufficient emphasis on the translation of efficacious interventions and activities into effective programmes (18). While the research imperatives posed by this challenge have been identified, with primacy given to the development of health systems research $(19,20)$, it is acknowledged that the latter is an underdeveloped field (4) that until recently has received little attention from epidemiologists.

\section{NEED OF EPIDEMIOLOGIC RESEARCH FOR IMPROVEMENT OF GLOBAL HEALTH}

In response to the above-mentioned challenges, epidemiologists should increasingly focus on research that addresses the implementation gap through involvement in research programmes that aim to identify, develop and evaluate new interventions for prevention and treatment of major poverty related health problems as well as to improve quality and equity in health and health care delivery. The term "new interventions" used here encompasses studies of efficacy and effectiveness of new as well as licensed medicinal products, such as existing drugs used in new combinations or on new indications. It also include research on interventions to modify behaviour, the physical and social environment as well as interventions to modify the health systems, e.g. evaluating the effect of employing new categories or combinations of health workers. For addressing these research issues, epidemiologic com- 
petence is crucial to validly measure the efficacy and effectiveness of new interventions for prevention, treatment and care. Several study designs are relevant.

There is a great need for randomized trials of new interventions as defined above. The trials can include phase III studies measuring the efficacy and effectiveness of new interventions that are envisaged to have a major positive public health impact. These trials can incorporate components enabling us to also measure cost-effectiveness of interventions as well as access to the interventions by marginalized population groups, thereby addressing equity. Further, such trials can contribute to identify bottlenecks for large-scale and equitable programmatic implementation in existing health systems or in health systems that can be improved within the current political and economic constraints.

Epidemiologists can contribute, not only in research for development and evaluation of interventions with specific drugs, vaccines or other specific interventions, but also through addressing interventions in relation to the organization and delivery of health services, e.g. governance, stewardship and knowledge management. Through community randomized trials interventions addressing health policy as well as sociocultural and personal factors that facilitate or inhibit implementation of effective technologies or practices can be evaluated. Epidemiologic designs can also be adapted to evaluate interventions that address infrastructural and organizational factors that facilitate or inhibit health system functioning.

In addition, epidemiologist should embark upon phase IV studies, i.e. studies that measure efficacy, effectiveness and/or cost-effectiveness as well as coverage of interventions that are already being implemen- ted in existing health programmes. Further, burden of disease studies are needed, incorporating detailed mapping of underlying and immediate causes, thereby enabling an estimation of not only the disease burden per se but also the extent to which specific modifiable risk factors contribute to this burden. Such measurements of causal associations are important for justifying and prioritizing large-scale interventions in public health programmes.

In general, there is a paucity of larger, long-term research initiatives in different local settings. Epidemiologists should join forces with other disciplines to evaluate comprehensive sets of interventions that work in concert (9) rather than parallel to each other, based on the most valid theories and current empirical knowledge.

\section{CONCLUSION}

Epidemiologic research is of utmost importance for reducing the global disease burden and reaching the four health-related millennium development goals. Priority should be given to intervention studies addressing risk factors for PRDs as well as critical health systems factors in low resource countries. Epidemiologists in high income countries are currently to a large extent involved in studies of risk factors and interventions of limited global public health importance. A shift of focus of epidemiologic research towards addressing health problems of major public health importance for disadvantaged population groups is needed. Through such a reorientation, epidemiology would gain a greater momentum in future research and development.

\section{REFERENCES}

1. Sanders D, Dovlo D, Meeus W, Lehmann U. Public health in africa. In: Beaglehole R, ed. Global Public Health: A New Era. Oxford: Oxford University Press, 2003: 135-55.

2. Joint Learning Initiative. Human Resources for Health: Overcoming the Crisis Report from the Joint Learning Initiative. Boston: Harvard University Press, 2004.

3. Gwatkin DR, Bhuiya A,Victora CG. Making health systems more equitable. Lancet 2004; 364 (9441): 127380 .

4. Travis P, Bennett S, Haines A, Pang T, Bhutta Z, Hyder AA, et al. Overcoming health-systems constraints to achieve the Millennium Development Goals. Lancet 2004; 364 (9437): 900-6.

5. Haines A, Sanders D. Building capacity to attain the Millennium Development Goals. Trans R Soc Trop Med Hyg 2005; 99 (10): 721-6.

6. Bryce J, Boschi-Pinto C, Shibuya K, Black RE. WHO estimates of the causes of death in children. Lancet 2005; 365 (9465): 1147-52.

7. Jones G, Steketee RW, Black RE, Bhutta ZA, Morris SS. How many child deaths can we prevent this year? Lancet 2003; 362 (9377): 65-71.

8. Lawn JE, Cousens S, Zupan Z. 4 million neonatal deaths: when? Where? Why? Lancet 2005; 365 (9462): 891-900.

9. Darmstadt GL, Bhutta ZA, Cousens S, Adam T, Walker N, de Bernis L. Evidence-based, cost-effective interventions: how many newborn babies can we save? Lancet 2005; 365 (9463): 977-88.

10. World Health Organization. The World Health Report 2005. Make every mother and child count. Geneva: World Health Organization, 2005. 
11. UNAIDS. Report on the global HIV/AIDS epidemic. Geneva and New York: UNAIDS, 2002.

12. Center for Economic and Policy Research. The scorecard on globalisation 1980-2000: Twenty years of diminished progress. Washington: CEPR, 2001.

13. UN General Assembly. "Declaration of Commitment on HIV/AIDS: "Global Crisis - Global Action"." UN Special Session on HIV/AIDS, 25-27 June 2001, New York.

14. UNAIDS. AIDS epidemic update. Geneva and New York: UNAIDS, 2004.

15. Pisani E, Garnett GP, Grassly NC, Brown T, Stover J, Hankins C, Walker N, Ghys PD. Back to basics in HIV prevention: focus on exposure. $B M J 2003 ; 326$ (7403): 1384-7.

16. Fylkesnes K, Musonda RM, Sichone M, Ndhlovu Z, Tembo F, Monze M. Declining HIV prevalence and risk behaviours in Zambia during the 1990s: evidence from national surveillance and population-based surveys. AIDS 2001; 15 (7): 907-916.

17. Fylkesnes K, Siziya S. A randomized trial on acceptability of voluntary HIV counselling and testing. Trop Med Int Health 2004; 9 (5): 566-572.

18. Sanders D, Labonte R, Baum F, Chopra M. Making research matter: a civil society perspective on health research. Bull World Health Organ 2004; 82 (10): 757-63.

19. The Mexico Statement on Health Research. Knowledge for Better Health: Strengthening Health Systems Ministerial Summit on Health Research, Mexico City, November 16-20, 2004.

20. Lancet (editorial). The Mexico Statement: strengthening health systems. Lancet 2004; 364 (9449): 1911-2. 